\title{
Short-course antibiotics for chemotherapy-induced febrile neutropaenia: retrospective cohort study
}

\author{
Nicola Seneviratne (ㄷ, ${ }^{1}$ Daniel Yeomanson, ${ }^{1}$ Robert Phillips ${ }^{2}$
}

${ }^{1}$ Haematology and Oncology, Sheffield Children's NHS Foundation Trust, Sheffield, UK ${ }^{2}$ Centre for Reviews and Dissemination, University of York, York, UK

\section{Correspondence to} Dr Daniel Yeomanson, Haematology and Oncology, Sheffield Children's NHS Foundation Trust, Sheffield S10 2TH, UK;

Daniel.Yeomanson@sch.nhs.uk

Received 12 July 2019

Revised 17 January 2020

Accepted 24 February 2020

Published Online First

17 March 2020
Check for updates

(C) Author(s) (or their employer(s)) 2020. No commercial re-use. See rights and permissions. Published by BMJ.

To cite: Seneviratne $\mathrm{N}$, Yeomanson D, Phillips R. Arch Dis Child

2020;105:881-885.

\section{ABSTRACT}

Background Recent research in febrile neutropaenia (FN) has focused on reducing the intensity of treatment for those thought to be at low risk of significant morbidity or mortality. This has not led to a reduced burden of treatment for either families or healthcare systems. An alternative approach is to discharge all patients who remain well after 48 hours of inpatient treatment, either with no ongoing treatment or with appropriate antibiotics if the cultures are positive. This paper aimed to demonstrate that this approach is safe. Methods Patients treated according to this approach in a single centre were reviewed retrospectively, with a random selection of patients from a 4-year period. Data were collected according to the Predicting Infectious Complications of Neutropenic sepsis in Children with Cancer dataset. In addition, all septic deaths over a 10 year period were reviewed in the same manner.

Results 179 episodes of FN were reviewed from 47 patients. In 70\% (125/179) of episodes, patients were discharged safely once 48-hour microbiology results were available, with only $5.6 \%(7 / 125)$ resulting in readmission in the 48 hours following discharge. There were no septic deaths in this cohort.

There were 11 deaths due to FN over the 10-year study period. Almost all patients were identified as severely unwell in the early stages of their final presentation or had a prolonged final illness.

Conclusion This paper indicates that the policy described provides a balance between safety and acceptability. Further work is needed to demonstrate non-inferiority and cost-benefit.

\section{INTRODUCTION}

Approximately 1600 children under 16 years of age are diagnosed with cancer in the UK each year. ${ }^{1}$ Most treatments involve chemotherapy, which results in myelosupression in a majority of cases, with a subsequent risk of severe infection.

Febrile neutropaenia (FN) is used to describe the clinical presentation of myelosuppressed patients with fever, although a wide range of temperature triggers and neutrophil thresholds have been used to identify such episodes. ${ }^{23}$

Advancements in supportive care for patients with cancer has seen the risk of death from FN fall from as high as $40 \%$ in the 1970 s to only $1 \%-3 \%$ more recently. ${ }^{3} 4$ Recognising that many children with FN remain well throughout admission and that clinically significant infections are rare, much work has been done to attempt to identify patients with 'low-risk' FN. ${ }^{5-9}$ This is important because management of FN episodes accounts for a considerable

\section{What is already known?}

- The mortality from febrile neutropaenia (FN) has fallen significantly, and many patients are at low risk of significant clinical consequences.

- Accurately identifying those at low risk of serious complications allows treatment to be deintensified.

- Current treatment models for 'low-risk' FN exclude significant numbers of patients.

\section{What this study adds?}

- Consideration of all patients for short-course treatment of FN appears safe.

- This approach should be considered alongside outpatient antibiotic regimens.

- Risk stratification 48 hours into an episode of FN reduces the likelihood of missing patients who have an overwhelming infection.

percentage of bed usage, which is expensive and inconvenient to patients and their families. Much of the management of FN takes place within shared care settings, using general paediatric beds outside Principle Treatment Centres (PTC). ${ }^{2}$

A number of strategies designed to reduce the duration of inpatient stay and/or early use of oral antibiotics have been evaluated as non-inferior to existing inpatient intravenous protocols. ${ }^{9-11}$

Although the details of antibiotic policies in these studies vary, they all examine the role of early step down to outpatient and/or oral antibiotics in a (variously) defined low-risk population. Such strategies have been recommended in international ${ }^{11}$ and UK national guidelines. ${ }^{12}$ In practice, whatever definition of low risk is adopted, up to three quarters of patients presenting with FN are excluded. ${ }^{25}$ These strategies therefore have a limited role to play in reducing the overall burden, to families and healthcare providers, of admission for $\mathrm{FN}$, as the majority of patients continue to receive a prolonged course of 'standard' inpatient intravenous antibiotic therapy.

This paper describes an alternative approach comprising an unstratified 48 hours of inpatient intravenous antibiotics, followed by discharge for all patients who have remained clinically well and have been afebrile for 24 hours, regardless of neutrophil count or underlying diagnosis. A positive blood culture does not preclude discharge of a well patient, with ongoing antimicrobial therapy. This is the shortest total duration of antibiotic 
therapy routinely used in paediatric oncology units in the $\mathrm{UK}^{3}$ and reduces antibiotic duration further than recommended in current UK NICE guidance. ${ }^{12}$ It is an approach previously identified as a strategy worthy of further investigation. ${ }^{4}$

We seek to demonstrate that this approach is safe and matches the desire of families to be in the hospital for as little time as possible.

\section{METHODS}

All patients studied were treated at a dedicated tertiary paediatric oncology unit according to the FN guideline, which has been in use with minor modifications for more than 15 years.

Under this policy, all patients with a documented temperature over $38.5^{\circ} \mathrm{C}$ (or with two temperatures at $>38.0^{\circ} \mathrm{C}$ more than 1 hour apart) are assessed on the ward. Assessment includes careful clinical review, full blood count and blood and urine cultures. Intravenous antibiotics (ceftazidime and gentamicin, unless known allergy or previous resistant organisms) are started immediately if the child is clinically unwell or has clinical signs consistent with invasive bacterial infection. Well children, without a clinical focus, are started on intravenous antibiotics if they have a neutrophil count of $<1.0 \times 10^{9} / \mathrm{L}$ or their neutrophil count is falling and anticipated to be $<1.0 \times 10^{9} / \mathrm{L}$ within 24 hours. The hospital does not have a shared care arrangement for FN; therefore, all patients are admitted to the PCT.

Once started on antibiotics, patients receive empirical antibiotics for 48 hours, while blood and urine culture results are awaited. A decision regarding ongoing management is made once the initial urine and 48-hour blood culture results are known. Those patients who are well, without fever for 24 hours and have negative cultures are discharged with no ongoing antibiotic treatment. If the cultures suggest infection, the patient may be discharged home on appropriate antibiotics (intravenous or oral), depending on the clinical and social situation. Patients who are deemed to be unwell or remain febrile continue inpatient treatment. The guideline is summarised in figure 1.

The guideline does not mandate what constitutes a well child. A consultant oncologist or haematologist ultimately decides whether an individual child goes home after the initial 48 hours of inpatient treatment or not. This enables factors such as social circumstances, the clinical status of the child, and the presence or absence of other toxicities to be taken into consideration.

All patients diagnosed with a malignancy (or treated with a haematopoietic stem cell transplant (HSCT)) between 2009 and 2012 were identified from the oncology department database. Each patient was allocated a randomly generated number and patients were entered into an Excel spreadsheet and sorted by their random number. The first 50 sets of patient notes were requested and examined in order. This was a pragmatic choice in order to balance practicality of data collection with the power of the results. For patients who had completed treatment or died, all episodes of FN were recorded. For patients undergoing active treatment at the time of data extraction, all episodes up to the time of collection were recorded. Multiple episodes of FN were recorded for individual patients.

A patient was considered to have been readmitted if they re-presented with fever within 48 hours of discharge of an admission for FN.

Data collected included age, tumour type, presence of central line, maximum temperature, microbiologically documented infections (MDIs), bloodstream infections (BSIs), intensive care unit (ICU) admission, organ support (including supplemental oxygen), duration of fever and duration of admission, and was based on the Predicting Infectious Complications of Neutropenic sepsis in Children with Cancer collaboration dataset. ${ }^{8}$

The main outcome measures were death, admission to ICU, presence of BSI, discharge following 48 hours of culture result and readmission within 48 hours of discharge with further fever.

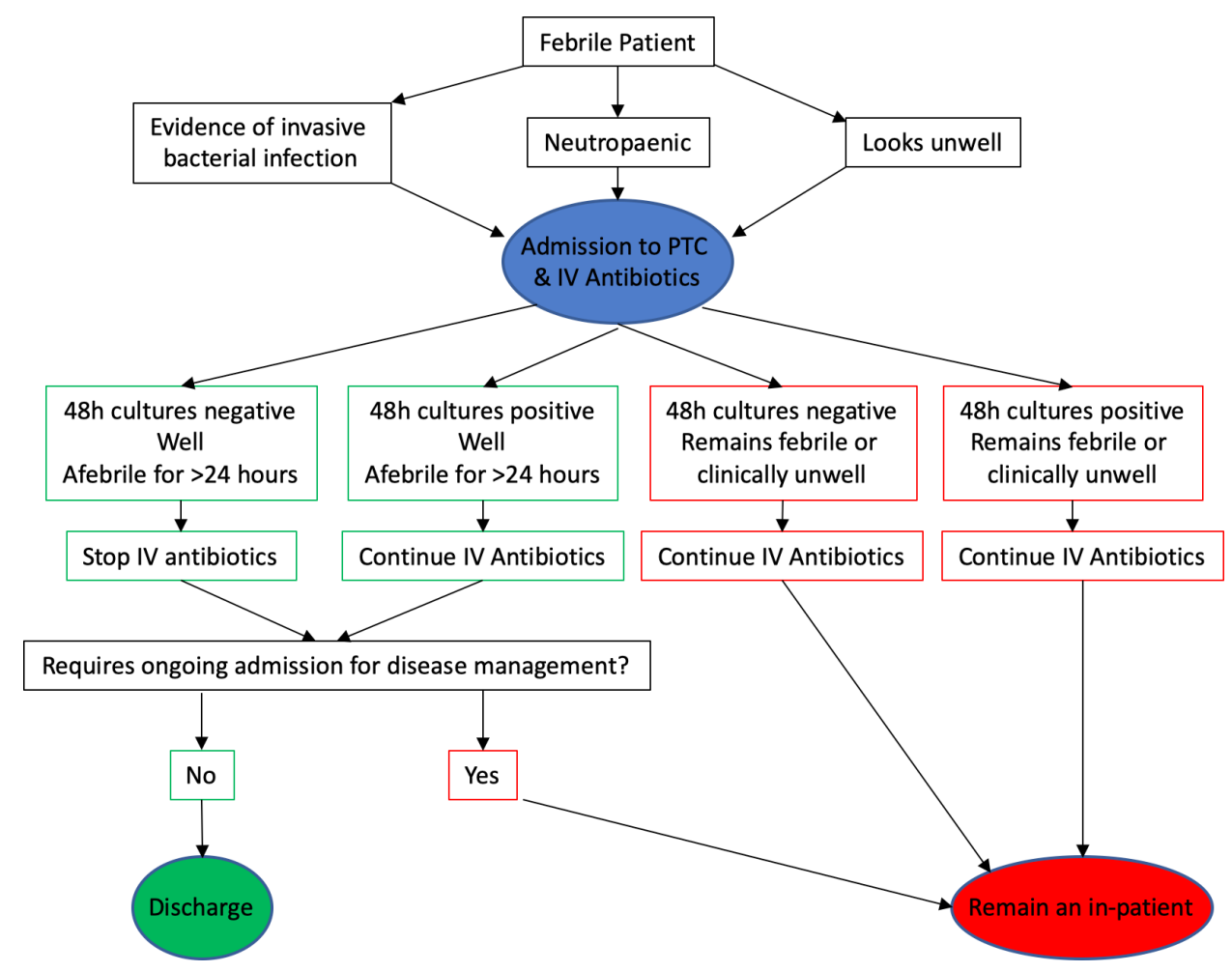

Figure 1 Summary of febrile neutropaenia guideline. PCT, principle treatment centre; IV, intravenous 
Table 1 Summary of underlying diagnoses

\begin{tabular}{lc}
\hline Underlying diagnosis & $\mathbf{n}(\%)$ \\
\hline Leukaemia & $22(47)$ \\
Brain tumour & $6(13)$ \\
Lymphoma & $3(6)$ \\
Other solid tumour & $16(34)$ \\
\hline
\end{tabular}

To ensure that random sampling did not miss the rarest but most important outcome of death, a second query was run to identify all patients who died of sepsis in a 10 -year period from 1 January 2002. These notes were reviewed and similar data were extracted.

\section{RESULTS}

There were 221 patients treated at the hospital for a malignancy between 2009 and 2012. Forty-seven patients with a malignant diagnosis between 2009 and 2012 had 179 episodes of FN during the study period. The notes from three other patients were either unavailable or they had not had an episode of FN. Twenty-six patients were male and 21 were female. The number of episodes per patient ranged from 1 to 12, with a median of 3 (IQR 1-5). The underlying diagnosis of the patients is given in table 1 .

Twelve per cent (22) of the episodes of FN occurred during treatment of relapsed or progressive disease. The majority of patients were undergoing standard intensity chemotherapy (55\% of episodes of $\mathrm{FN}$ ) with a further $9 \%$ occurring in those who had had a HSCT. The intensity of chemotherapy given prior to episodes of FN split by eligibility for early discharge is shown in table 2 .

In this cohort, there were no septic deaths. Four episodes involved admission to the ICU. Organ support (including supplemental oxygen) without admission to the ICU was required in 18 $(10 \%)$ cases. There were 64 (36\%) MDIs and 26 (15\%) episodes with BSI. The outcomes for the episodes of FN are shown in table 3 .

The median duration of recorded fever was 2 days (range nil-28 days), and the median admission duration was 3 days (range 1-31 days, IQR 2.0-5.25).

Overall, 125 of 179 (70\%) episodes of FN met the criteria for discharge following the initial culture result. Twenty-six patients had a proven bloodstream infection, with 11 (42\%) of these discharged early on antibiotics. Readmission within 48 hours of conclusion of the primary episode was documented in seven episodes (5.6\%). One patient was initially discharged on appropriate antibiotics for a coagulase-negative Staphylococcus (CONS) infection and re-presented unwell with an Escherichia coli-positive blood culture, for which the patient required a

\begin{tabular}{lcl}
\hline Table 2 & Chemotherapy intensity & \\
\hline & $\begin{array}{l}\text { Eligible for discharge } \\
\text { once culture result is } \\
\text { available } \\
\mathbf{n}(\%)\end{array}$ & $\begin{array}{l}\text { Not eligible for } \\
\text { discharge once culture } \\
\text { result is available } \\
\mathbf{n}(\%)\end{array}$ \\
Chemotherapy intensity & 0 \\
\hline Neutropaenia due to disease & $2(2)$ & $14(26)$ \\
Low & $47(38)$ & $37(69)$ \\
Standard & $62(50)$ & $3(6)$ \\
HSCT & $14(11)$ & 54 \\
\hline Total & 125 & \\
\hline
\end{tabular}

HSCT, haematopoietic stem cell transplant.

\begin{tabular}{llllll} 
Table 3 & \multicolumn{5}{l}{ Outcome of episodes of febrile neutropaenia } \\
& Death & $\begin{array}{l}\text { ICU } \\
\text { admission }\end{array}$ & $\begin{array}{l}\text { Organ } \\
\text { support }\end{array}$ & $\begin{array}{l}\text { No additional } \\
\text { support }\end{array}$ & Total (\%) \\
\hline No MDI & 0 & 2 & 10 & 102 & $114(64)$ \\
All MDIs & 0 & 2 & 8 & 54 & $64(36)$ \\
BSIs & 0 & 1 & 4 & 21 & $26(15)$ \\
Total (\%) & $0(0)$ & $4(2)$ & $18(10)$ & $156(87)$ & $179(100)$
\end{tabular}

$\mathrm{BSI}$, bloodstream infection; ICU, intensive care unit; MDI, microbiologically documented infection.

brief admission to ICU for inotropic support. The other patients who required re-admission were not unwell, and some were presumed or confirmed to have viral infections. A summary of patient outcomes is shown in figure 2 .

There were 16 patient deaths in the department related to infection over the 10-year period. Five of these patients were excluded as they did not have cancer (2 patients) or were not neutropenic during the episode that led to their death (3 patients). Of the remaining 11 septic deaths, 5 were due to a prolonged infective process, and 5 presented severely unwell and died despite aggressive resuscitation and ICU admission. One patient deteriorated and died while receiving appropriate outpatient intravenous antibiotics for a coagulase-negative Staphylococcus (CONS) bacteraemia. It is unclear what caused this deterioration, as microbiology at the time of deterioration was negative.

\section{DISCUSSION}

There have been numerous attempts to stratify an individual's risk of severe infection at presentation, ${ }^{4-9} 11{ }^{13-16}$ so that those deemed to be at low risk of serious complications (eg, need for organ support, ICU admission and death) undergo treatment at a reduced intensity and duration. However, clinical application

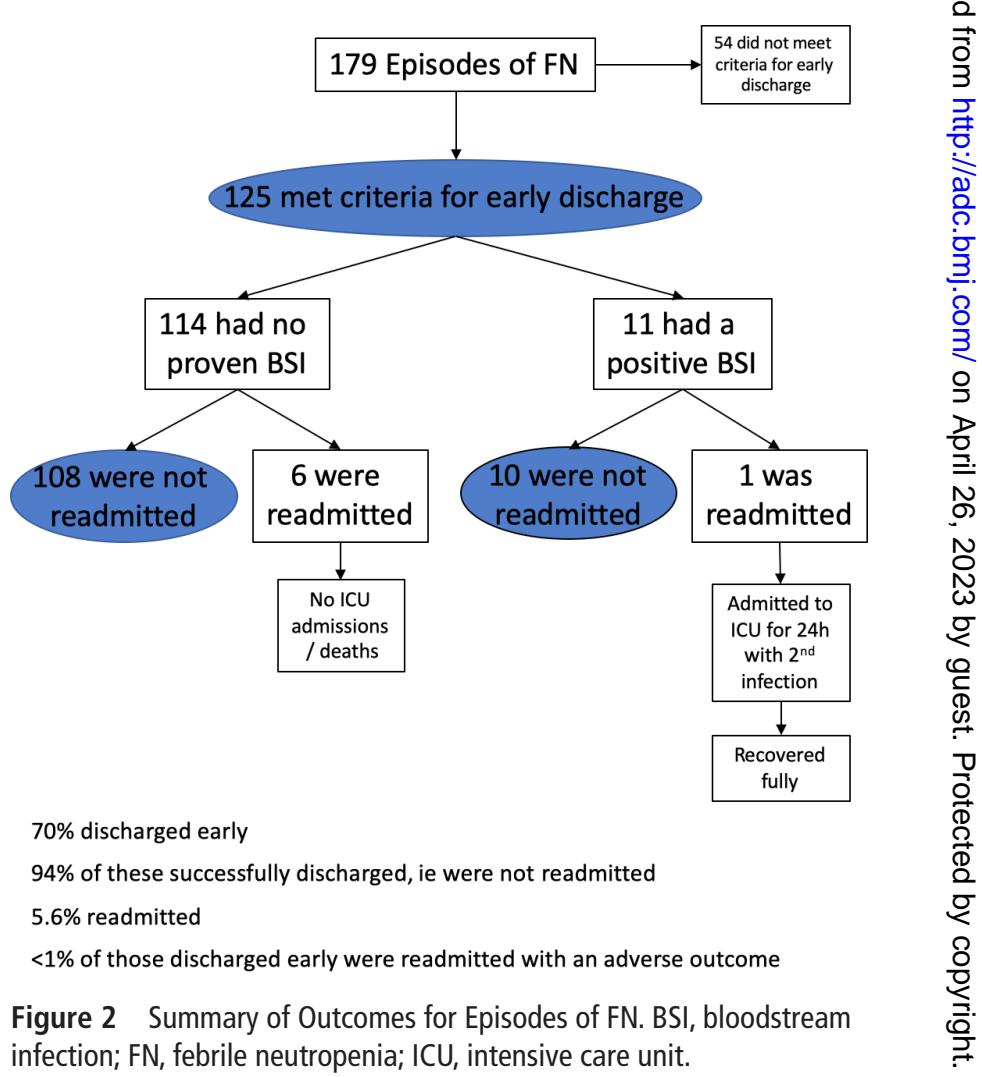


of risk stratification is not straightforward, with some having complex algorithms requiring senior input at the time of presentation. None of these formal risk prediction strategies are sufficiently sensitive to exclude all those patients who go on to develop a significant infection. It has been demonstrated that for some systems, reassessment in the first 24 hours after presentation improves the sensitivity of the stratification. ${ }^{10}$

Once a patient has been deemed to be at low risk of complications of FN, a variety of approaches have been described to reduce the intensity of treatment, and each of these has its own advantages and disadvantages. ${ }^{4}$ Early discharge on intravenous antibiotics reduces the length of hospital stay but requires either community nursing support or daily outpatient visits. Ambulatory treatment with intravenous antibiotics from the outset has minimal inpatient stay but again requires support from community nursing or daily hospital visits and has the potential for early deterioration outside the hospital. Early discharge on oral antibiotics reduces duration of hospital stay and also allows a period of observation and the opportunity for culture results to be available; however, they require the child to be able to tolerate oral antibiotics. Finally, outpatient treatment with oral antibiotics from the outset enables all treatment to be provided at home, but not only is it limited by the ability to tolerate oral therapy, but it also risks deterioration, which must be recognised by parents, and has been associated with a $14 \%$ readmission rate. ${ }^{15}$

The outcomes of the strategy of universal aggressive inpatient treatment and early discontinuation of antibiotics are promising. Patients were discharged once 48 hours of blood culture results were available, without further treatment or review in $70 \%$ of episodes of FN. This compares to up to $75 \%$ of patients being excluded from low risk stratification. Those with ongoing symptoms or proven infection underwent continuing treatment, as an inpatient or outpatient based on clinical and social considerations.

Although a small proportion of those children discharged returned with fever in the 48 hours following discharge, these children were not catastrophically unwell.

When deciding the optimal management strategy in paediatric practice, in addition to medical outcomes and cost implications, it is important to incorporate family/parental preferences. A survey of parental views showed that $>75 \%$ of parents preferred at least some of the treatment to be hospital based, ${ }^{16}$ with focus group studies confirming a general preference for admissions to be as short as possible without compromising safety. ${ }^{17}$ Our strategy acknowledges this preference as all patients stay in hospital for at least 48 hours. Parents have the reassurance that at discharge, their child is deemed by the specialist to be well. Beyond aligning with parental wishes, reduced hospitalisation leads to a reduction in nosocomial infections, frees up resources to deliver chemotherapy and improves the quality of lived experience while undergoing treatment. ${ }^{1819}$

An important objective in the management of $\mathrm{FN}$ is the prevention of death. Although not a common outcome, it is clearly important that any management strategy does not increase the risk of this. Our review of all septic deaths demonstrated that the vast majority of patients were either seriously unwell at initial presentation or had prolonged admissions with repeated infection, multiple changes to antibiotics and multiorgan involvement. The only exception to this was a patient who was readmitted severely unwell 5 days into a course of intravenous antibiotics. It was presumed that the deterioration was due to a second organism causing severe sepsis, although there was no positive microbiology. There was potential for the deterioration to have been identified earlier had they been receiving the antibiotics as an inpatient. However, it is recognised that serious complications can occur late and may be preceded by apparently less severe symptoms, ${ }^{6}$ and that prophylactic hospitalisation does not prevent all septic deaths. ${ }^{19}$

\section{LIMITATIONS}

The data were collected retrospectively from a 4-year period, although not all episodes of FN were included. This leads to the potential for missing data, creating bias. To collect the data prospectively would have been impractical in a single centre, over a short time period, due to the relatively small number of patients and the rarity of adverse events. Random selection of patients, together with the unambiguous primary endpoints (ICU admission, readmission within 48 hours and death) and a specific analysis of septic deaths strengthen the findings and go some way to overcoming the limitations of retrospective analysis, as does our clear description of the completeness of data. The assessment of safety from the 50-note review is underpowered but strengthened by the assessment of a further group of septic deaths over a longer time period. While the sensitivity of blood culture alone in detecting significant infection has been questioned, ${ }^{20}$ its use, alongside clinical features of absent or controlled infection, makes this a clinically pragmatic diagnostic gold standard.

This protocol has been successfully applied in the oncology unit at a dedicated tertiary children's hospital. Care is consultant led and supported by a cohort of experienced paediatric oncology nurses. In addition, there is close support from a consultant microbiologist and a dedicated oncology pharmacist, who are key team members. Further work is needed to determine whether it can be safely transferred to other settings where there is less consistent experience of caring for children with cancer.

\section{CONCLUSION}

$\mathrm{FN}$ is a common cause for admission among paediatric oncology patients, with both financial and psychosocial costs. For most patients, the risk of significant morbidity or mortality from an episode of FN is low. The antibiotic policy described in this paper provides an appropriate balance between safety and acceptability, and should be considered for wider use, ideally as one arm of a multicentre randomised non-inferiority trial of step-down therapy for FN. Any such study should include a robust cost-benefit analysis and examine patient/parent satisfaction with the various treatment approaches.

Acknowledgements Many thanks to our data managers, Janet Williams and Emma Eaton, for maintaining a detailed database of patient outcomes, allowing us to identify patients who died of sepsis over the study period. Thanks also to Fiona Payne, David King and StaceyKillick, who assisted with data extraction from clinical notes.

Contributors NS made substantial contributions to the interpretation and discussion of findings and drafted, reviewed and revised the manuscript. DY had the initial study idea; contributed to the conception, design and data collection; and also reviewed and revised the manuscript for important intellectual content. $\mathrm{RP}$ contributed to the interpretation of the data and the structure of the report, in addition to reviewing the manuscript for important intellectual content.

Funding The authors have not declared a specific grant for this research from any funding agency in the public, commercial or not-for-profit sectors.

Competing interests None declared.

Patient consent for publication Not required.

Provenance and peer review Not commissioned; externally peer reviewed.

Data availability statement Data are available upon reasonable request. Please contact the corresponding author if you require access to the data used in this paper.

ORCID iD

Nicola Seneviratne http://orcid.org/0000-0003-0788-1288 


\section{REFERENCES}

1 Incidence of childhood cancer in the UK. Available: https://www.cclg.org.uk/CSOIR/ Incidence-of-childhood-cancer-in-the-UK [Accessed 7 Jul 2019].

2 Herd F, Bate J, Chisholm J, et al. Variation in practice remains in the UK management of paediatric febrile neutropenia. Arch Dis Child 2016;101:410-1.

3 Phillips B, Selwood K, Lane SM, et al. Variation in policies for the management of febrile neutropenia in United Kingdom children's cancer Study Group centres. Arch Dis Child 2007;92:495-8.

4 Chisholm JC, Dommett R. The evolution towards ambulatory and day-case management of febrile neutropenia. Br J Haematol 2006;135:3-16.

5 RackoffWR, Gonin R, Robinson C, et al. Predicting the risk of bacteremia in childen with fever and neutropenia. J Clin Oncol 1996;14:919-24.

6 Ammann RA, Bodmer N, Hirt A, et al. Predicting adverse events in children with fever and chemotherapy-induced neutropenia: the prospective multicenter SPOG 2003 FN study. J Clin Oncol 2010;28:2008-14.

7 Brack E, Bodmer N, Simon A, et al. First-Day step-down to oral outpatient treatment versus continued standard treatment in children with cancer and low-risk fever in neutropenia. A randomized controlled trial within the multicenter SPOG 2003 FN study. Pediatr Blood Cancer 2012:59:423-30.

8 Phillips RS, Sutton AJ, Riley RD, et al. Predicting infectious complications in neutropenic children and young people with cancer (IPD protocol). on behalf of the PICNICC collaboration. Syst Rev 2012;1:8

9 Phillips RS, Wade R, Lehrnbecher T, et al. Systematic review and meta-analysis of the value of initial biomarkers in predicting adverse outcome in febrile neutropenic episodes in children and young people with cancer. BMC Med 2012;10:6.

10 Manji A, Beyene J, Dupuis LL, et al. Outpatient and oral antibiotic management of low-risk febrile neutropenia are effective in children--a systematic review of prospective trials. Support Care Cancer 2012;20:1135-45.
11 Phillips RS, Lehrnbecher T, Alexander S, et al. Updated systematic review and metaanalysis of the performance of risk prediction rules in children and young people with febrile neutropenia. PLoS One 2012;7:e38300.

12 . Available: http://www.nice.org.uk/guidance/cg151

13 Alexander SW, Wade KC, Hibberd PL, et al. Evaluation of risk prediction criteria for episodes of febrile neutropenia in children with cancer. J Pediatr Hematol Oncol 2002;24:38-42.

14 Phillips RS, Sung L, Ammann RA, et al. Predicting microbiologically defined infection in febrile neutropenic episodes in children: global individual participant data multivariable meta-analysis. Br J Cancer 2016;114:623-30.

15 Morgan JE, Cleminson J, Atkin K, et al. Systematic review of reduced therapy regimens for children with low risk febrile neutropenia. Support Care Cancer 2016;24:2651-60

16 Diorio C, Martino J, Boydell KM, et al. Parental perspectives on inpatient versus outpatient management of pediatric febrile neutropenia. J Pediatr Oncol Nurs 2011;28:355-62.

17 Phillips RS, Depani S, Morgan JE. What do families want to improve in the management of paediatric febrile neutropenia during anti-cancer treatment? BMJ Paediat Open 2019;3:e000398.

18 Morgan JE, Phillips B, Stewart LA, et al. Quest for certainty regarding early discharge in paediatric low-risk febrile neutropenia: a multicentre qualitative focus group discussion study involving patients, parents and healthcare professionals in the UK. BMJ Open 2018:8:e020324.

19 Sung L, Aplenc R, Alonzo TA, et al. Effectiveness of supportive care measures to reduce infections in pediatric AML: a report from the children's Oncology Group. Blood 2013;121:3573-7.

20 Dien Bard J, McElvania TeKippe E. Diagnosis of bloodstream infections in children. J Clin Microbiol 2016:54:1418-24. 